\title{
Inventory, diversity and therapeutic uses of medicinal plants in the Tiaret Mountains (western Algeria)
}

\author{
Nouar Belgacem "*, Hasnaoui Okkacha', Maamar Benchohra² \& Tir Elhadj ${ }^{3}$ \\ ${ }^{1}$ Laboratory of Ecology and Natural Ecosystem Management, University of Tlemcen, Algeria \\ ${ }^{2}$ Faculty Natura and Life sciences, University of Tiaret, Algeria \\ ${ }^{3}$ Faculty of Nature and Life and Earth Sciences and the Universes, University of Tlemcen, Algeria \\ *Corresponding author, email: nouar.belkacem@hotmail.fr
}

\begin{abstract}
This study is devoted to the floristic (systematic, biology, chorology) and therapeutic (treated uses and diseases) analysis of medicinal plants in the Tiaret Mountains (western Algeria). The floristic inventory allowed us to identify 108 medicinal plants belonging to 41 families, including the dominance of the Asteraceae, Lamiaceae, Fabaceae and Orchidaceae with $(15.7 \%, 7.4 \%, 5.6 \%, 5.6 \%)$ respectively. Also we identified 3 endemic, 4 rare and 8 protected species. The biological spectrum of these species is characterized by a high presence of the Therophytes (38\%) followed by the Phanerophytes (20.4\%). In biogeographical terms, Mediterranean biogeographical species are the most represented and make up more than half of the medicinal flora (52.8\%). These plants are mainly used in order of importance in the treatment of the digestive tract, cardiovascular and dermal diseases with percentages of $18.6 \%$, $10.7 \%, 7.5 \%$.
\end{abstract}

KEY WORDS Floristic analysis; Inventory; Medicinal plants; Therapeutic uses; Tiaret Mountains.

Received 30.04.2020; accepted 15.10.2020; published online 15.02.2021

\section{INTRODUCTION}

Medicinal plants are still a source of medical care in developing countries (Rebbas \& Bounar, 2014). A large majority (70-80\%) of people in Africa consult traditional medicine practitioners (TPMs) for health care (Cunningham, 1993). Algeria is a typical example and one of the countries with a long medical tradition and a knowledge of medicinal plants (Baba-Aissa, 1991), some of these plants are used by residents living in the area and are marketed by herbalists (Benaissa et al., 2018). But according to (Rebbas et al., 2012), the use of conventional medicine has led to the neglect of these ancestral practices, which risk being forgotten. However, this country remains poorly explored, even though it encompasses considerable natural re- sources distributed in different ecosystems and has considerable floristic diversity (Miara et al., 2019). In this context, the aim of this study is to identify and enhance the medicinal plants of the Tiaret mountains by their floristic analysis and their uses in traditional medicine.

\section{MATERIAL AND METHODS}

\section{Study area}

Our study area is an integral part of the Tiaret Mountains, western Algeria (Fig. 1), which are located in the north of the department (Wilaya) and embracing the city of Tiaret in the south, they are between 700 and over 1200 meters high. The bio- 
climate goes from semi-arid to cool winters, however, this bioclimatic area overflows towards the arid at low altitudes and the subhumid at the highest peaks (Miara et al., 2016). The soil of the region is characterized by a sandy texture, which is most often decarbonated. This would come from the dominant geological nature of the region (Miara et al., 2012).

\section{Sampling}

Two hundred floristic surveys were carried in the optimum period of vegetation (March-June) during the years 2015-2018 following stratified sampling according to Gounot (1969) by listing all species present with a minimum surface area of 100 $\mathrm{m}^{2}$, the latter seems sufficient for the plant formations studied (Dahmani, 1997).

The identification of taxa was made from the flora of Quèzel \& Santa (1962-1963) updated by the index of Dobignard \& Chatelain (2010-2013). Biological types of species are performed using the notion of Raunkiaer (1934).

\section{Medicinal plants}

To select medicinal species and their traditional uses we have used the works of medicinal plants in the region of Algeria (Baba-Aissa, 1991; Beloued, 2005) and other various scientific articles on ethnobotanical studies carried in the country (Rebbas et al., 2012; Boudjelal et al., 2012; Miara et al., 2013; Hachemi et al., 2013; Cherma \& Gharzouli, 2015).

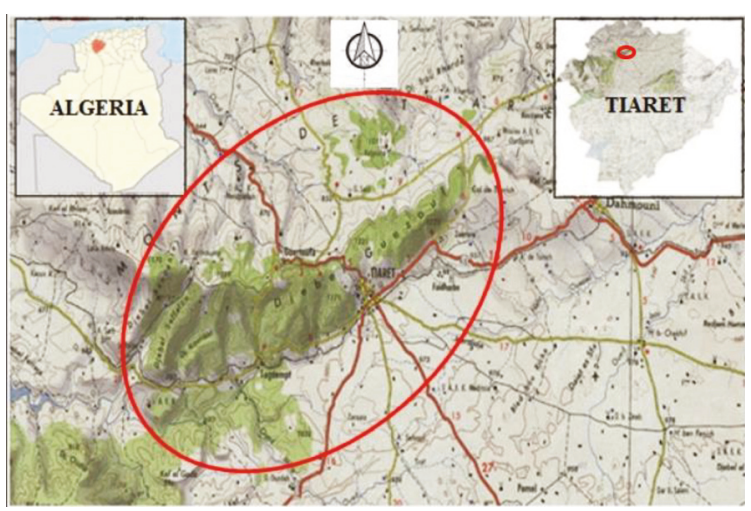

Figure 1. Location of the study area: Tiaret Mountains (western Algeria).

\section{RESULTS AND DISCUSSION}

\section{Floristic composition}

The analysis of the inventoried flora (300 species in total) provided a list of 108 medicinal plant divided into 85 genera and 41 botanical families (Fig. 2). The richest families in spices are Asteraceae $(17 ; 15.7 \%)$, Lamiaceae $(8 ; 7.4 \%)$, Fabaceae and Orchidaceae $(6 ; 5.6 \%)$, Boraginaceae and Plantaginaceae $(5 ; 4.6 \%)$, Apiaceae and Cistaceae $(4$; $3.7 \%)$. The rest of the families are poorly represented with percentages that vary between them $(3,2$ and 1 species; $2.8 \%, 1.9 \%$ and $0.9 \%$ respectively).

\section{Biological types}

The classification of species by biological types was marked by the dominance of the Therophytes (41; 38\%) followed by Phanerophytes $(22 ; 20.4 \%)$, Hemicryptophytes $(18 ; 16.7 \%)$ then Chamaephytes $(16 ; 14.8 \%)$ and finally the Geophytes $(11 ; 10.2 \%)$ (Fig. 3).

The high representation of Therophytes reveals high anthropogenic pressure (Daget 1980), but these annual plants are widely used by the population and have a socio-economic role (Hachemi et al., 2013). Phanerophytes represented by trees and shrubs often sempervirens have a significant percentage explained by their presence over time by their roots, branches and leaves (Miara et al., 2013).

\section{Biogeographic types}

Based on the analysis of the chorological types, we observe the predominance of Mediterranean biogeographical species $(57 ; 52.8 \%)$, one of the characteristics of the typical Mediterranean flora (Miara et al., 2017). Secondly, come the western Mediterranean elements $(11 ; 10.2 \%)$, followed by the Euro-Mediterranean $(9 ; 8.3 \%)$, the Cosmopolitans $(7 ; 6.5 \%)$, Eurasians $(6 ; 5.6 \%)$, then the Mediterranean Canaries, North African Endemics, Europeans, Ibero-Mauritanian, Atlantic Mediterranean and Sub Mediterranean with the same number of species $(2 ; 1.9 \%)$. The remainder of the species represent low participation (one species), but contribute to the diversity and richness of the plant potential of the study area (Fig. 4). 


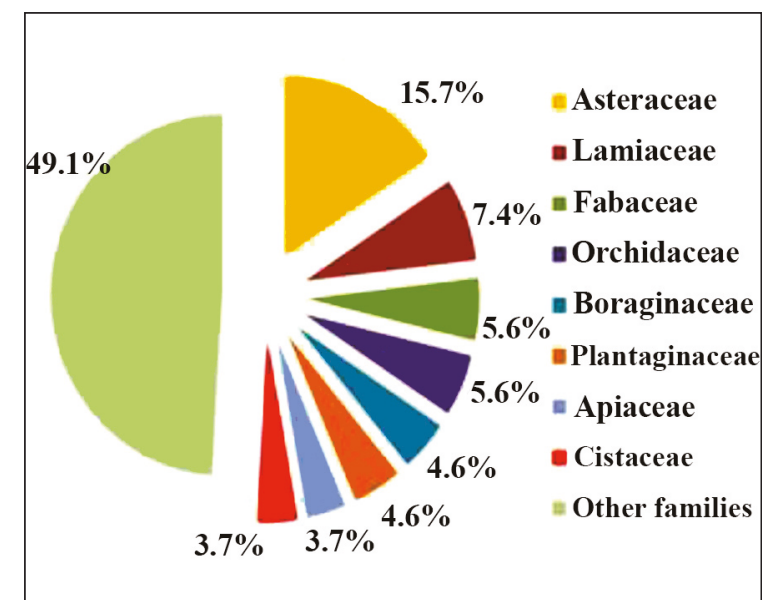

Figure 2. Composition of medicinal plants by family.

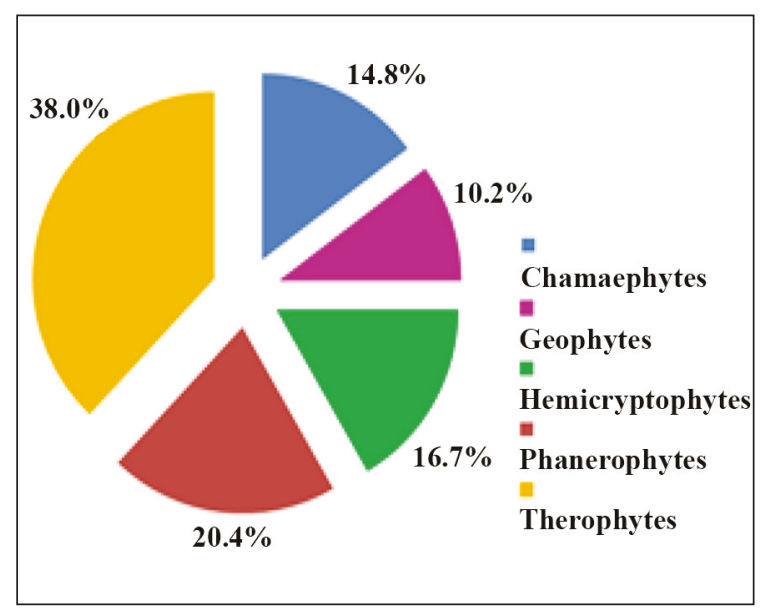

Figure 3. Biological spectrum of medicinal plants.

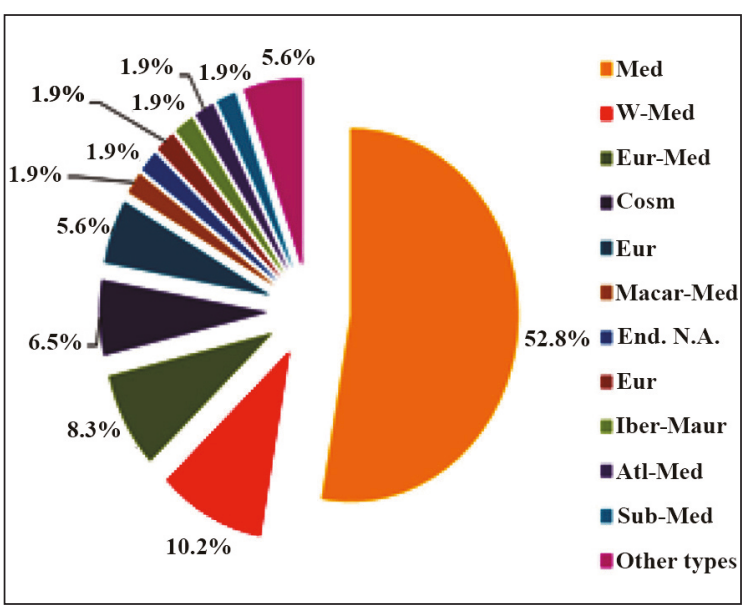

Figure 4. Chorological spectrum of medicinal plants.

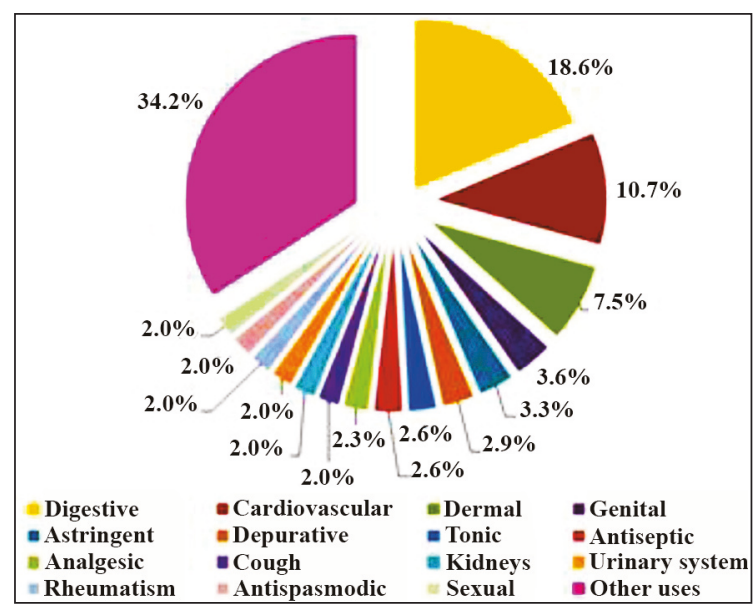

Figure. 5. Diseases treated by medicinal plants.

\section{Endemic, rare and protected plants}

The medicinal flora of the Tiaret Mountains is characterized by the presence of two (2) endemic species of the North African (End N.A) Helianthemum ledifolium subsp. apertum (Pomel) Raynaud ex Greuter \& Burdet and Thymus ciliatus (Desf.) Benth. subsp. ciliatus and one (1) endemic species Algeria-Tunisia (End Alg-Tun) Origanum vulgare subsp. glandulosum (Desf.) Ietsw.

The analysis of species rarity showed the existence of one (1) quite rare (QR) species Orchis papilionacea L. and three (3) rare (R) species Blackstonia perfoliata subsp. grandiflora (Viv.) Mayor, Phillyrea angustifolia L. and Quercus suber L.

According to Executive Decree No. 12-03 of 10 Safar 1433 corresponding to 4 January 2012 fixing the list of protected non-cultivated plant species, eight (08) protected species have been inventoried: Bellis prostrata Pomel, Gagea algeriensis Chabert, Juniperus oxycedrus L., Orchis olbiensis Reut. ex Gren, Orchis papilionacea L., Otocarpus virgatus Durieu, Pistacia atlantica Desf. and Tetraclinis articulata (Vahl) Mast.

These species are grouped in Table 1.

\section{Therapeutic uses}

The analysis of the types of diseases treated by the inventoried medicinal plants shows that the majority of these plants are used in the treatment of the digestive tract $(18.6 \%)$, notably stomach and 
colon colic, intestinal disease, etc., cardiovascular diseases such as hypertension, heart disease, etc., comes secondly with $10.7 \%$, while dermal disease care such as (skin irritations and eczema) is recommended by several species and comes thirdly $(7.5 \%)$. These results are in line with the observations of Miara et al. (2013), Benarba et al. (2015), Chermat \& Gharzouli (2015), Ouelbani et al. (2016), and Bouasla \& Bouasla (2017). Genital diseases are treated at a rate of $3.3 \%$, while many species have astringentes properties $(3.6 \%)$, other depuratives $(3 \%)$, tonic and antiseptic $(2.6 \%)$ and even analgesic $(2.3 \%)$.

Other diseases treated are: cough, kidney diseases, urinary system diseases, rheumatism, spasms and sexual disability in men with the same percentage $(2 \%)$ each. In addition, a low recommendation of these plants (less than $2 \%$ ) for the care of various diseases such as: diabetes, liver diseases, respiratory diseases, tonsillitis, bronchitis, migraine and forms a percentage of $34.2 \%$ in total (Fig. 5, see also Table 2).

\section{CONCLUSIONS}

The inventory of medicinal plants in the Tiaret Mountains shows a great diversity with immense therapeutic virtues for the treatment of common diseases and certain chronic diseases such as diabetes and hypertension.

So this work could provide useful information on each of the these plants that will make it possible to use their in the field of pharmacology.

For this purpose, plant chemical and pharmacological studies should be undertaken in the future to create an economic activity around the use of these plants in an organized and respectful environment to the preservation of endemic, rare and protected species.

\begin{tabular}{|c|c|c|c|}
\hline Taxa & Endemism & Rarity & $\begin{array}{l}\text { Protection } \\
\text { (ED) }\end{array}$ \\
\hline Bellis prostrata Pomel & & & $\mathrm{P}$ \\
\hline Blackstonia perfoliata subsp grandiflora (Viv.) Maire & & $\mathrm{R}$ & \\
\hline Gagea algeriensis Chabert & & & $\mathrm{P}$ \\
\hline $\begin{array}{l}\text { Helianthemum ledifolium subsp. apertum (Pomel) Raynaud ex } \\
\text { Greuter \& Burdet }\end{array}$ & End N.A & & \\
\hline Juniperus oxycedrus L. & & & $\mathrm{P}$ \\
\hline Orchis olbiensis Reut. ex Gren. & & & $\mathrm{P}$ \\
\hline Orchis papilionacea $\mathrm{L}$. & & QR & $\mathrm{P}$ \\
\hline Origanum vulgare subsp. glandulosum (Desf.) Ietsw & End Alg-Tun & & \\
\hline Otocarpus virgatus Durieu & & & $\mathrm{P}$ \\
\hline Phillyrea angustifolia L. & & $\mathrm{R}$ & \\
\hline Pistacia atlantica Desf. & & & $\mathrm{P}$ \\
\hline Quercus suber L. & & $\mathrm{R}$ & \\
\hline Tetraclinis articulata (Vahl) Mast & & & $\mathrm{P}$ \\
\hline Thymus ciliatus (Desf.) Benth. subsp. ciliatus & End. N.A & & \\
\hline
\end{tabular}

Table 1. List of endemic, rare and protected medicinal plants from the study area: Tiaret Mountains (western Algeria). End N.A: Endemic North. African; End Alg-Tun : Endemic Algeria-Tunisia; QR: Quite rare; R: Rare. P: Protected; ED: Executive Decree. 


\begin{tabular}{|c|c|c|c|}
\hline TAXA & $\begin{array}{l}\text { BIO- } \\
\text { LOGY }\end{array}$ & $\begin{array}{l}\text { CHORO- } \\
\text { TYPE }\end{array}$ & THERAPEUTIC USE \\
\hline \multicolumn{4}{|l|}{ ANACARDIACEAE } \\
\hline Pistacia atlantica Desf. & $\mathrm{PH}$ & N.A-SICILY & Astringent, stomach diseases, coughs and colds \\
\hline Pistacia lentiscus L. & $\mathrm{PH}$ & MED & $\begin{array}{l}\text { Colon and stomach colic, stomach ulcer and varicose } \\
\text { veins }\end{array}$ \\
\hline Pistacia terebinthus $\mathrm{L}$. & $\mathrm{PH}$ & MED & Genital diseases, colon \\
\hline \multicolumn{4}{|l|}{ APIACEAE } \\
\hline $\begin{array}{l}\text { Daucus carota subsp maximus } \\
\text { (Desf.) Batt. }\end{array}$ & $\mathrm{HE}$ & MED & $\begin{array}{l}\text { Visual acuity, jaundice, in various states of nervous } \\
\text { exhaustion, also for pinworm in children }\end{array}$ \\
\hline Eryngium campestre L. & $\mathrm{HE}$ & EUR-MED & $\begin{array}{l}\text { Disorders of the bladder and kidneys, urinary affections, } \\
\text { cough and bronchitis }\end{array}$ \\
\hline Ferula communis L. & $\mathrm{CH}$ & MED & Migraine \\
\hline Thapsia garganica $\mathrm{L}$ & $\mathrm{HE}$ & MED & Rheumatic pains, in cases of pulmonary diseases \\
\hline \multicolumn{4}{|l|}{ APOCYNACEAE } \\
\hline Nerium oleander L. & $\mathrm{PH}$ & MED & Dermatology (scab, eczema), for bowel disorders \\
\hline \multicolumn{4}{|l|}{ ARECACEAE } \\
\hline Chamaerops humilis $\mathrm{L}$ & $\mathrm{CH}$ & W-MED & $\begin{array}{l}\text { Diabetes, decreases the level of total cholesterol and } \\
\text { triglycerides }\end{array}$ \\
\hline \multicolumn{4}{|l|}{ ASPARAGACEAE } \\
\hline Asparagus acutifolius $\mathrm{L}$. & GE & MED & Stomachic and diuretic, cardiovascular diseases \\
\hline Asparagus albus L. & GE & W-MED & Jaundice and rheumatism, appetizer and stomachic \\
\hline Drimia maritima (L.) Stearn & GE & $\begin{array}{l}\text { MACAR- } \\
\text { MED }\end{array}$ & Heart failure and reduced kidney activity \\
\hline \multicolumn{4}{|l|}{ ASTERACEAE } \\
\hline Anacyclus clavatus (Desf.) Pers & $\mathrm{TH}$ & EUR-MED & Skin and difficult digestion \\
\hline Anacyclus pyrethrum (L.) Link & $\mathrm{HE}$ & $\begin{array}{l}\text { IBERO- } \\
\text { MAUR }\end{array}$ & $\begin{array}{l}\text { Used mostly externally, toothache, paralyzed tongue and } \\
\text { to excite abundant salivation }\end{array}$ \\
\hline Bellis annua L. subsp. аппиа & TH & MED & Analgesic, anti-inflammatory, diuretic \\
\hline Bellis sylvestris $\mathrm{L}$. & $\mathrm{TH}$ & MED & Analgesic, anti-inflammatory, diuretic \\
\hline Calendula arvensis (Vaill.) L. & $\mathrm{TH}$ & MED & $\begin{array}{l}\text { Depurative, emmenagogues, antispasmodic and } \\
\text { stimulating }\end{array}$ \\
\hline $\begin{array}{l}\text { Calendula suffruticosa subsp. } \\
\text { boissieri Lanza }\end{array}$ & TH & MED & $\begin{array}{l}\text { Antiseptic, constituents are fungicidal, antibacterial and } \\
\text { antiviral, healing action }\end{array}$ \\
\hline Centaurea calcitrapa $\mathrm{L}$. & $\mathrm{HE}$ & MED & Tonic, fabrifuges, diuretic \\
\hline Centaurea pullata $\mathrm{L}$. & $\mathrm{TH}$ & MED & Increase the pressure \\
\hline Cichorium intybus L. & $\mathrm{HE}$ & COSM & $\begin{array}{l}\text { Slightly tonic, stomachic, depurative, regulating } \\
\text { cholagogue of the gastrointestinal tract }\end{array}$ \\
\hline $\begin{array}{l}\text { Echinops spinosus subsp. } \\
\text { eu.spinosus Greuter }\end{array}$ & $\mathrm{HE}$ & S-MED-SAH. & Labor pains, abortion, neuralgia \\
\hline $\begin{array}{l}\text { Helichrysum stoechas (L.) } \\
\text { Moench }\end{array}$ & $\mathrm{CH}$ & W-MED & Helminth infections, anti-dandruff \\
\hline $\begin{array}{l}\text { Pallenis spinosa (L.) Cass. subsp. } \\
\text { spinosa }\end{array}$ & $\mathrm{CH}$ & EUR-MED & Antidiabetic, anti-inflammatory \\
\hline Reichardia picroides (L.) Roth & TH & MED & A little diuretic \\
\hline Scolymus maculatus L. & $\mathrm{HE}$ & MED & Liver diseases and intestines \\
\hline Senecio vulgaris L. & TH & SUBCOSM & Emmenagogue \\
\hline Silybum marianum L. & $\mathrm{HE}$ & MED & $\begin{array}{l}\text { Appetizers, tonic, fabrifugal and resolutive, diseases of } \\
\text { the liver, spleen, jaundice and chronic constipation }\end{array}$ \\
\hline $\begin{array}{l}\text { Sonchus tenerrimus L. subsp } \\
\text { tenerrimus }\end{array}$ & $\mathrm{TH}$ & MED & Depurative \\
\hline
\end{tabular}




\begin{tabular}{|c|c|c|c|}
\hline TAXA & $\begin{array}{l}\text { BIO- } \\
\text { LOGY }\end{array}$ & $\begin{array}{l}\text { CHORO- } \\
\text { TYPE }\end{array}$ & THERAPEUTIC USE \\
\hline \multicolumn{4}{|l|}{ BORAGINACEAE } \\
\hline Anchusa azurea Mill. & TH & EUR-MED. & Antitumor, anti-inflammatory, wound-healing \\
\hline Borago officinalis L. & $\mathrm{TH}$ & W-MED & $\begin{array}{l}\text { As temperamental in burning, bilious and eruptive fever, } \\
\text { in engorgements of the liver }\end{array}$ \\
\hline Cynoglossum cheirifolium L. & $\mathrm{TH}$ & MED & Astringent, diarrheal, soothing \\
\hline Cynoglossum creticum Miller & $\mathrm{HE}$ & MED & Astringent, diarrheal, soothing \\
\hline Echium plalltagineum $\mathrm{L}$. & TH & MED & Diuretic \\
\hline \multicolumn{4}{|l|}{ BRASSICACEAE } \\
\hline $\begin{array}{l}\text { Capsella bursa-pastoris (L.) } \\
\text { Medik. }\end{array}$ & $\mathrm{TH}$ & MED & $\begin{array}{l}\text { Astringent, against diarrhea and dysentery, uterine } \\
\text { haemorrhages, to women whose periods are repeated } \\
\text { too frequently or for too long }\end{array}$ \\
\hline Lobularia maritima (L.) Desv. & TH & MED & Febrifuge \\
\hline Sinapis arvensis L. & TH & $\begin{array}{l}\text { PALEO } \\
\text { TEMP }\end{array}$ & Increases the secretion of the intestinal mucosa \\
\hline \multicolumn{4}{|l|}{ CAMPANULACEAE } \\
\hline Campanula rapunculus $\mathrm{L}$. & TH & EUR-MED. & $\begin{array}{l}\text { Antiseptic, tonic, used especially for the treatment of } \\
\text { tonsillitis }\end{array}$ \\
\hline \multicolumn{4}{|l|}{ CAPRIPHOLIACEAE } \\
\hline Lomelosia stellata $\mathrm{L}$. & $\mathrm{TH}$ & W-MED & Treatment of fevers for children \\
\hline Lonicera implexa Aiton & $\mathrm{PH}$ & MED. & Astringent, sudorific, diuretic \\
\hline \multicolumn{4}{|l|}{ Caryophylaceae } \\
\hline Paronychia argentea Lam. & $\mathrm{TH}$ & MED & $\begin{array}{l}\text { Diuretic, aseptic and slightly spasmodic. Treatment of } \\
\text { inflammation of the urinary tract, kidneys and gall } \\
\text { bladder }\end{array}$ \\
\hline Stellaria media (L.) Vill. & TH & COSM & Skin irritations, severe itching, eczema, ulcers \\
\hline \multicolumn{4}{|l|}{ CISTACEAE } \\
\hline $\begin{array}{l}\text { Cistus creticus subsp. eriocepha- } \\
\text { lus (Viv.) Greuter \& Burdet }\end{array}$ & $\mathrm{CH}$ & MED & Aperitif, used as an aphrodisiac \\
\hline Cistus monspeliensis L. & $\mathrm{CH}$ & MED & Aperitif, used as an aphrodisiac \\
\hline Cistus salvifolius L. & $\mathrm{CH}$ & MED & Aperitif, used as an aphrodisiac \\
\hline $\begin{array}{l}\text { Helianthemum ledifolium subsp. } \\
\text { apertum (Pomel) Raynaud ex } \\
\text { Greuter \& Burdet }\end{array}$ & $\mathrm{TH}$ & END N.A & Lactation, irregular cycle \\
\hline CONVOLVULACEAE & & & \\
\hline Convolvulus althaeoides L. & $\mathrm{HE}$ & MED. & Antispasmodic, stomachic, tonic, purgative \\
\hline \multicolumn{4}{|l|}{ CRASSULACEAE } \\
\hline Umbilicus horizontalis (Guss.) DC. & $\mathrm{HE}$ & ATL-MED & Healing, emollient \\
\hline \multicolumn{4}{|l|}{ CUPRESSACEAE } \\
\hline Cupressus sempervirens L. & $\mathrm{PH}$ & MED & $\begin{array}{l}\text { Stimulant, diuretic, stomach tonic, pulmonary antiseptic } \\
\text { and depurative, disorders of the venous system, in the } \\
\text { treatment of varicose veins }\end{array}$ \\
\hline Juniperus oxycedrus L. & $\mathrm{PH}$ & $\begin{array}{l}\text { IBERO- } \\
\text { MAUR }\end{array}$ & $\begin{array}{l}\text { Stimulating, diuretic, stomach tonic, pulmonary anti- } \\
\text { septic and depurative, against skin diseases (eczema, } \\
\text { psoriasis ...) and as a vermifuge }\end{array}$ \\
\hline Tetraclinis articulata (Vahl) Mast & $\mathrm{PH}$ & MED & $\begin{array}{l}\text { Intestinal and respiratory infections. In external use is } \\
\text { applied to the umbilical wound of newborns for scarring. } \\
\text { Dental care, digestive disorders, nausea, respiratory } \\
\text { problems, fever }\end{array}$ \\
\hline
\end{tabular}




\begin{tabular}{|c|c|c|c|}
\hline TAXA & $\begin{array}{l}\text { BIO- } \\
\text { LOGY }\end{array}$ & $\begin{array}{l}\text { CHORO- } \\
\text { TYPE }\end{array}$ & THERAPEUTIC USE \\
\hline \multicolumn{4}{|l|}{ ERICACEAE } \\
\hline Arbutus unedo L. & $\mathrm{PH}$ & MED & $\begin{array}{l}\text { Antiseptic and very astringent, against diarrhea, } \\
\text { dysentery, urinary tract inflammation }\end{array}$ \\
\hline \multicolumn{4}{|l|}{ EUPHORBIACEAE } \\
\hline $\begin{array}{l}\text { Euphorbia helioscopia L. subsp. } \\
\text { eu-helioscopia }\end{array}$ & $\mathrm{TH}$ & AS-EUR & Purgative, vesicant \\
\hline $\begin{array}{l}\text { Anthyllis vulneraria subsp. maura } \\
\text { (Beck) Maire }\end{array}$ & $\mathrm{TH}$ & EUR-MED & $\begin{array}{l}\text { Astringent, vulnerable, depurative and slightly laxative. } \\
\text { Against the intestinal and stomach laziness and vomiting } \\
\text { of children }\end{array}$ \\
\hline Astragalus ramosus L. & TH & MED & Emollient, softening, galactagogue \\
\hline $\begin{array}{l}\text { Calicotome spinosa (L.) Link } \\
\text { subsp. spinosa }\end{array}$ & $\mathrm{CH}$ & W. MED & Various episodes of intoxication and as hypoglycemic. \\
\hline $\begin{array}{l}\text { Coronilla scorpioides (L.) W.D.J. } \\
\text { Koch }\end{array}$ & TH & MED & $\begin{array}{l}\text { Leaves: purgative; Seeds: asthma, heart conditions, } \\
\text { purgative, scorpion sting. }\end{array}$ \\
\hline Lotus ornithopodioides L. & TH & MED & Astringent, vulnerary \\
\hline $\begin{array}{l}\text { Scorpiurus muricatus subsp. } \\
\text { sulcatus (L.) TheIl. }\end{array}$ & TH & MED & Scorpion sting \\
\hline \multicolumn{4}{|l|}{ FAGACEAE } \\
\hline $\begin{array}{l}\text { Quercus coccifera L. subsp. } \\
\text { coccifera }\end{array}$ & $\mathrm{PH}$ & W. MED & Treatment of dysentery \\
\hline $\begin{array}{l}\text { Quercus ilex subsp. ballota } \\
\text { (Desf.) Samp }\end{array}$ & $\mathrm{PH}$ & MED & Urinary disorders of children \\
\hline Quercus suber L. & $\mathrm{PH}$ & W-MED & $\begin{array}{l}\text { Used as a haemostatic and healing in wound care. } \\
\text { Antidiarrheal and in the treatment of diseases of the } \\
\text { stomach and colon }\end{array}$ \\
\hline \multicolumn{4}{|l|}{ GENTIANACEAE } \\
\hline $\begin{array}{l}\text { Blackstonia perfoliata subsp. } \\
\text { grandiflora (Viv.) Maire }\end{array}$ & $\mathrm{TH}$ & MED & Febrifuge, stomachic, tonic \\
\hline $\begin{array}{l}\text { Centaurium erythraea subsp. } \\
\text { suffruticosum (Salzm. ex Griseb.) } \\
\text { Greuter }\end{array}$ & TH & EUR-MED & $\begin{array}{l}\text { Against dysentery, flatulence, hemorrhoids, mucous } \\
\text { fevers. Also to increase the pressure }\end{array}$ \\
\hline \multicolumn{4}{|l|}{ GERANIACEAE } \\
\hline Geranium purpureum Vill. & $\mathrm{TH}$ & COSM & Gastric pain and gallbladder disease \\
\hline \multicolumn{4}{|l|}{ LAMIACEAE } \\
\hline Lavandula stoechas L. & $\mathrm{CH}$ & MED & Stomach and pectorals pains, also known as diuretic \\
\hline Marrubium vulgare L. & $\mathrm{HE}$ & COSM & $\begin{array}{l}\text { Liver diseases, allergies, and gallbladder care, fights } \\
\text { pelvic pain and coughing }\end{array}$ \\
\hline $\begin{array}{l}\text { Origanum vulgare subsp. } \\
\text { glandulosum (Desf.) Ietsw }\end{array}$ & HE & $\begin{array}{l}\text { END ALG- } \\
\text { TUN }\end{array}$ & $\begin{array}{l}\text { Used as a herbal tea to cure several diseases such as: } \\
\text { rheumatism, cough, colds and digestive disorders }\end{array}$ \\
\hline Rosmarinus officinalis L. & $\mathrm{CH}$ & MED & $\begin{array}{l}\text { Stimulants, antispasmodic, diuretic, cholagogue and } \\
\text { vermifuge. Calm stomach colic and flu }\end{array}$ \\
\hline Salvia verbenaca (L.) Briq. & $\mathrm{HE}$ & ATL-MED & Stimulant, tonic, antiseptic \\
\hline Teucrium pseudochamaepitys L. & TH & W-MED & $\begin{array}{l}\text { Used as a tea to treat digestive disorders, spasms } \\
\text { and colic }\end{array}$ \\
\hline Teucrium thymoides Pomel. & $\mathrm{CH}$ & EUR-MED & Vomiting and pinworms \\
\hline $\begin{array}{l}\text { Thymus ciliatus (Desf.) Benth. } \\
\text { subsp. ciliatus }\end{array}$ & $\mathrm{CH}$ & END. N.A & $\begin{array}{l}\text { Depurative, influenza, cough, cold, abdominal } \\
\text { meteorism and diseases of the endocrine glands }\end{array}$ \\
\hline \multicolumn{4}{|l|}{ MALVACEAE } \\
\hline Malva sylvestris $\mathrm{L}$. & $\mathrm{TH}$ & AS-EUR & $\begin{array}{l}\text { Headache, drowsiness, urine retention and kidney } \\
\text { disease }\end{array}$ \\
\hline
\end{tabular}




\begin{tabular}{|c|c|c|c|}
\hline TAXA & $\begin{array}{l}\text { BIO- } \\
\text { LOGY }\end{array}$ & $\begin{array}{l}\text { CHORO- } \\
\text { TYPE }\end{array}$ & THERAPEUTIC USE \\
\hline \multicolumn{4}{|l|}{ OLEACEAE } \\
\hline Olea europea L. subsp. europaea & $\mathrm{PH}$ & MED & $\begin{array}{l}\text { Gum disease and high blood pressure. Against the flu, } \\
\text { cardiovascular problems and heals the skin. Diuretic, } \\
\text { antidiabetic }\end{array}$ \\
\hline Phillyrea angustifolia $\mathrm{L}$. & $\mathrm{PH}$ & MED & Treatment of fever \\
\hline \multicolumn{4}{|l|}{ ORCHIDACEAE } \\
\hline Ophrys fusca Link. & GE & MED & $\begin{array}{l}\text { Tubers are used to treat the problems of sexual disability } \\
\text { of men }\end{array}$ \\
\hline Ophrys lutea (Cav.) Gouan & GE & MED & \\
\hline Ophrys speculum Link & GE & MED & \\
\hline Ophrys tenthredinifera Willd. p.p. & GE & MED & \\
\hline Orchis olbiensis Reut. ex Gren & GE & AS-EUR & \\
\hline $\begin{array}{l}\text { Orchis papilionacea } \mathrm{L} .=\text { Ana- } \\
\text { camptis papilionacea }(\mathrm{L} .) \text { R.M. } \\
\text { Bateman, Pridgeon \& M.W. Chase }\end{array}$ & GE & MED & \\
\hline \multicolumn{4}{|l|}{ PAPAVERACEAE } \\
\hline Fumaria officinalis L. & TH & EUR & $\begin{array}{l}\text { Diuretic and cholagogue, debility of digestive tract, } \\
\text { abdominal congestions, jaundice, scars and cutaneous } \\
\text { affections }\end{array}$ \\
\hline Papaver rhoeas $\mathrm{L}$ & $\mathrm{TH}$ & COSM & $\begin{array}{l}\text { Antispasmodic, sedative, recommended for insomniacs } \\
\text { and against eye inflammation }\end{array}$ \\
\hline \multicolumn{4}{|l|}{ PINACEAE } \\
\hline Pinus halepensis Mill. & $\mathrm{PH}$ & MED & Expectorant, balsamic, mild diuretic, antiseptic, astringent \\
\hline Pinus pinea $\mathrm{L}$. & $\mathrm{PH}$ & MED & $\begin{array}{l}\text { Respiratory diseases, urinary disorders, stomach ulcers } \\
\text { and weakness. In external use balsamic, antiseptic and } \\
\text { antirheumatic }\end{array}$ \\
\hline \multicolumn{4}{|l|}{ PLANTAGINACEAE } \\
\hline Globularia alypum subsp alypum & $\mathrm{CH}$ & MED & Antiparasitic, stomachic, cure for diabetes and colon \\
\hline Plantago afra $\mathrm{L}$. & TH & MED & $\begin{array}{l}\text { Constipation, diarrhea, inflammatory bowel disease, } \\
\text { reduces cholesterol and blood glucose levels, prevent } \\
\text { coronary heart disease }\end{array}$ \\
\hline Plantago albicans $\mathrm{L}$. & $\mathrm{HE}$ & MED & Against diarrhea, poultices in the care of wounds \\
\hline Plantago bellardii All & $\mathrm{TH}$ & AS-EUR & Laryngitis, diarrhea \\
\hline $\begin{array}{l}\text { Plantago coronopus L. subsp. } \\
\text { coronopus }\end{array}$ & $\mathrm{HE}$ & MED & Softening, astringent, emollient \\
\hline \multicolumn{4}{|l|}{ POACEAE } \\
\hline $\begin{array}{l}\text { Ampelodesmos mauritanicus } \\
\text { (Poir.) Durand \& Schinz }\end{array}$ & $\mathrm{CH}$ & W-MED & Kidney stones and gallbladder \\
\hline Avena sterilis $\mathrm{L}$. & TH & COSM & Softening, anti-asthenic, emollient \\
\hline $\begin{array}{l}\text { Macrochloa tenacissima }(\mathrm{L} .) \\
\text { Kunth = Stipa tenacissima } \mathrm{L} .\end{array}$ & GE & MED & Pressure problems \\
\hline \multicolumn{4}{|l|}{ POLYGONACEAE } \\
\hline Rumex bucephalophorus L. & TH & EUR & $\begin{array}{l}\text { Disorders due to a high level of toxins in the body, } \\
\text { against constipation }\end{array}$ \\
\hline \multicolumn{4}{|l|}{ PRIMULACEAE } \\
\hline $\begin{array}{l}\text { Lysimachia arvensis }(\mathrm{L} .) \mathrm{U} . \\
\text { Manns \& Anderb. = Anagallis } \\
\text { arvensis } \mathrm{L} .\end{array}$ & TH & COSM. & Diuretic, depurative, expectorant and hepatic \\
\hline
\end{tabular}




\begin{tabular}{|c|c|c|c|}
\hline TAXA & $\begin{array}{l}\text { BIO- } \\
\text { LOGY }\end{array}$ & $\begin{array}{l}\text { CHORO- } \\
\text { TYPE }\end{array}$ & THERAPEUTIC USE \\
\hline \multicolumn{4}{|l|}{ RANUNCULACEAE } \\
\hline Clematis flammula $\mathrm{L}$ & $\mathrm{PH}$ & MED & Analgesic, rubefiant, vesicant \\
\hline \multicolumn{4}{|l|}{ RESEDACEAE } \\
\hline Reseda alba L. subsp. alba & $\mathrm{TH}$ & EUR-AS & Diarrhea, colic and digestive intoxications \\
\hline Reseda collina Müll. Arg. & $\mathrm{HE}$ & MED. & Sudorific \\
\hline Reseda luteola L. & TH & EUR-AS & Sudorific \\
\hline \multicolumn{4}{|l|}{ RHAMNACEAE } \\
\hline $\begin{array}{l}\text { Rhamnus lycioides subsp. } \\
\text { oleoides L. }\end{array}$ & $\mathrm{PH}$ & W-MED & Hepatitis and jaundice \\
\hline Ziziphus lotus (L.) Desf. & $\mathrm{PH}$ & MED & For all diseases, especially lung diseases \\
\hline \multicolumn{4}{|l|}{ ROSACEAE } \\
\hline Crataegus monogyna Jacq & $\mathrm{PH}$ & EUR-MED & $\begin{array}{l}\text { Anxiety, insomnia, dizziness, hypertension, cardiac and } \\
\text { nervous disorders also to calm colic and diarrhea. In } \\
\text { external use it is useful in the case of sore throat }\end{array}$ \\
\hline \multicolumn{4}{|l|}{ SANTALACEAE } \\
\hline Osyris alba $\mathrm{L}$. & $\mathrm{CH}$ & MED. & Aphrodisiac, alopecia \\
\hline \multicolumn{4}{|l|}{ SMILACACEAE } \\
\hline Smilax aspera $\mathrm{L}$. & $\mathrm{PH}$ & MED & $\begin{array}{l}\text { Rheumatism, asthma, skin diseases, kidney and urinary } \\
\text { infections }\end{array}$ \\
\hline \multicolumn{4}{|l|}{ TAMARICACEAE } \\
\hline Tamarix gallica $\mathrm{L}$ & $\mathrm{PH}$ & N. TROP & Liver and diabetes \\
\hline \multicolumn{4}{|l|}{ THYMELAEACEAE } \\
\hline Thymelaea hirsuta Endl. & $\mathrm{CH}$ & MED & Softening for the hair. Treats cough and constipation \\
\hline \multicolumn{4}{|l|}{ XANTHORRHOEACEAE } \\
\hline $\begin{array}{l}\text { Asphodelus microcarpus } \\
\text { Salzm. et Viv. }\end{array}$ & GE & $\begin{array}{l}\text { MACAR- } \\
\text { MED }\end{array}$ & Otitis, rheumatism and dental pain \\
\hline
\end{tabular}

Table 2. List of medicinal plants from the study area: Tiaret Mountains (western Algeria).

\section{ACKNOWLEDGEMENTS}

The authors thank the DGRSDT for the financial support of this work.

\section{REFERENCES}

Baba-Aissa F., 1991. Les plantes médicinales en Algérie. In: Bouchéne et Ad Diwan (Co-Ed), Alger, $189 \mathrm{pp}$

Beloued A., 2005. Les plantes médicinales d'Algérie. Ed. Office des publications universitaires (OPU), Alger, $284 \mathrm{pp}$.

Benaissa M., El Haitoum A. \& Hadjadj K., 2018. Floristic and medical diversity interest of Djebel Aissa national park (Ksour Montains, Algeria). Malaysian
Journal of Fundamental and Applied Sciences, 14, 2: 303-306. https://doi.org/10.11113/mjfas. v14n2.1032

Benarba B., Belabid L., Righi K., Bekkar A.A., Elouissi M., Khaldi A. \& Hamimed A., 2015. Ethnobotanical study of medicinal plants used by traditional healers in Mascar (North West of Algeria). Journal of Ethnopharmacology. https://dx.doi.org/10.1016/j.jep. 2015.09.030

Bouasla A. \& Bouasla I., 2017. Ethnobotanical survey of medicinal plants in northeastern of Algeria. Phytomedicine. https://dx.doi.org/10.1016/j.phymed. 2017.09.007

Boudjelal A., Henchiri C., Sari M., Sarri D., Hendel N., Benkhaled A. \& Ruberto G., 2013. Herbalists and wild medicinal plants in M'Sila (North Algeria): An ethnopharmacology survey. Journal of Ethnopharmacology, 148: 395-402. 
Chermat S. \& Gharzouli R., 2015. Ethnobotanical Study of Medicinal Flora in the North East of Algeria - An Empirical Knowledge in Djebel Zdimm (Setif). Journal of Materials Science and Engineering A 5 (1-2): 50-59. https://dx.doi.org/0.17265/2161-6213/2015.12.007

Cunningham A.B., 1993. African medicinal plants: Setting priorities at the interface between conservation and primary healthcare, to People and Plants Inintiative. Division of Ecological Sciences. UNESCO. 7 Place de Fontenoy. 75352 Paris CEDEX 07 SP, France, $50 \mathrm{pp}$.

Daget P., 1980. Les types biologiques en tant que stratégie adaptative (les thérophytes). In: Babault R., Blandin P. \& J. Meyer (Eds.), Recherches d'écologie théorique. Les stratégies adaptatives, Maloine.

Dobignard A. \& Chatelain C., 2010-2013. Index synonymique de la flore d'Afrique du Nord, 1-5. Genève

Hachemi N., Hasnaoui O., Bouazza M., Benmehdi I. \& Medjati N., 2013. The Therophytes Aromatic and Medicinal Plants of the Southern Slopes of the Mountains of Tlemcen (Western Algeria) Between Utility and Degradation. Research Journal of Pharmaceutical, Biological and Chemical Sciences, 4: 1194 1203.

Miara M.D., Ait Hammou M. \& Hadjadj Aoul S., 2013. Phytothérapie et taxonomie des plantes médicinales spontanées dans la région de Tiaret (Algérie). Phytothérapie Springer-Verlag France, 13 pp. https:// dx.doi.org/10.1007/s10298-013-0789-3.

Miara M.D., Ait Hammou M., Hadjadj Aoul S., Dahmani W., Negadi M., Rebbas K., Bounar R. \& Smaili T., 2016. Notes sur les thérophytes dans les monts de Tiaret (massif de Guezoul-Algérie occidentale). Revue Ècologie-Environnement, 12: 17-24.

Miara M.D., Hadjadj Aoul S. \& Ait Hammou M., 2012. Analyse phytoécologique et syntaxonomique des groupements végétaux dans le Massif de GuezoulTiaret (N-O Algérie). Bulletin de la Société Botanique du Centre-Ouest - Nouvelle Série, 43: 279-316.

Miara M.D., Ait Hammou M., Hadjadj-Aoul S., Rebbas K. \& Bendif H., 2017. Diversité floristique des milieux forestiers et préforestiers de l'Atlas Tellien occidental de Tiaret (N-O Algérie). Revue électronique annuelle de la Société botanique du Centre-OuestEvaxiana, 4: 201-225.

Miara M.D., Bendif H., Rebbas K., Rabah B., Ait Hammou M. \& Maggi F., 2019. Medicinal plants and their traditional uses in the highland region of Bordj Bou Arreridj (Northeast Algeria), Journal of Herbal Medicine, 16: 100262. https://doi.org/10.1016/j. hermed.2019.100262

Quézel P. \& Santa S., 1962-1963. Nouvelle Flore de l'Algérie et des régions désertiques méridionales, Thom. I-II. C.N.R.S., Paris, 1170 pp.

Raunkiaer C., 1934. The Life Forms of Plants and Statistical Plant Geography, Oxford Clarendon Press, 1934.

Ouelbani R., Bensari S., Nardjes Mouas T. \& Khelifi D., 2016. Ethnobotanical investigations on plants used in folk medicine in the regions of Constantine and Mila (Northeast of Algeria), Journal of Ethnopharmacology, 194: 196-218. https://dx.doi.org/10.1016/ j.jep.2016.08. 016

Rebbas K. \& Bounar R., 2014. Etudes floristique et ethnobotanique des plantes médicinales de la région de M'Sila (Algérie). Phytothérapie, Springer-Verlag France, 8 pp. https://doi.org/10.1007/s10298-0140872-4.

Rebbas K., Bounar R., Gharzouli R., Ramdani M., Djellouli M. \& Alatou D., 2012. Plantes d'intérêt médicinale et écologique dans la région d'Ouanougha (M'Sila). Phytotherapie, 6: 1-12. 